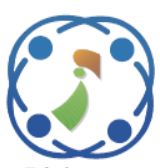

\title{
A Novel Image Watermarking Scheme Based on Wavelet Transform and Genetic Algorithm
}

\author{
Nagraju Naik Mood ${ }^{1 *} \quad$ Venkata Subbareddy Konkula ${ }^{2}$ \\ ${ }^{1}$ Department of Electronics and Communication Engineering, CMR College of Engineering \& Technology (A), \\ Kandlakoya, Medchal, Hyderabad, Telangana, India \\ ${ }^{2}$ Department of Electronics and Communication Engineering, Hyderabad, Telangana, India \\ * Corresponding author's Email: nagarajunaik1976@cmrcet.org
}

\begin{abstract}
This paper proposes a Secure, robust and intelligent watermarking approach based on Redundant Wavelet Transform, Singular Value Decomposition and Genetic Algorithm. Here the robustness. The proposed watermarking scheme accomplishes RWT and SVD for feature extraction and the GA for optimization. Further this approach proposes a signature embedding mechanism by which the watermarked image will be more secure. The Genetic Algorithm proposed in this approach adopts intelligent property and the signature process adopts more security. Further the combination of RWT and SVD adopts the robustness to the proposed approach. Numerous experiments are conducted over the proposed approach and the performance is evaluated through the performance metrics such as Peak Signal to Noise ratio (PSNR) and Normalized Correlation (NC). The obtained experimental results demonstrates that the proposed approach is superior compared to conventional approach and is able to provide efficient resistance over Gaussian noise, salt \& pepper noise, median filtering, cropping, rotation, contrast enhancement, scaling and Histogram Equalization attacks.
\end{abstract}

Keywords: Image watermarking, Redundant wavelet transform, Singular value decomposition, Genetic algorithm, Attack, PSNR, NC, SSIM.

\section{Introduction}

Currently, with rapid growth of the Internet and digital media, large amounts of media data are transmitted through the Internet due to its convenience and amazing speed. Digital media content and copyright are confronted with great challenges. Many researchers are aware of the importance of copyright protection, image authentication, and so forth, and they have great interest in applying watermarking scheme into digital multimedia for copyright protection $[1,2]$. One of the most important branches in digital watermarking community is robust watermarking, which aims at achieving robustness, imperceptibility, and high security simultaneously [3]. According to the domain used for embedding, the current schemes of digital watermark are basically classified into two types [4]: (i) Spatial domain; (ii) Transform domain.
Spatial domain methods embed a watermark via modifications to the pixel values of an original image. Transform-domain schemes embed a watermark via modifications to the coefficients of the corresponding transformed-domain image of an original image. The transform domain involves discrete Fourier transform (DFT), discrete cosine transform (DCT), and discrete wavelet transform (DWT), etc.

This paper proposes a novel image watermarking technique based on Redundant Wavelet Transform (RWT) and Genetic Algorithm (GA). Compared to the conventional wavelet transforms, the RWT reduces the information loss in the extracted watermark. Since, the RWT is shift invariant, the watermark extraction leads to more accurate and effective watermark at the extraction stage. Further the GA is used for the optimization of watermarking constant, alpha. To ensure the security 
of the watermark, a novel signature is embedded in the watermarked image by which the attacker can't acquire the information even though he/she attacks the watermarked image. This is the main advantage of the proposed approach which was not focused in the earlier approaches mostly. To show the efficiency of proposed approach, various images were processed for testing. Finally, the robustness of proposed approach was tested by applying various attacks on the watermarked image.

The rest of the paper is organized as follows: section 2 illustrates the complete details about the related work. Section 3 illustrates the details about the indents of proposed watermarking technique such as Genetic Algorithm, Discrete wavelet transform, Redundant Wavelet transform and the singular value decomposition. The complete detail of proposed watermarking methodology is illustrated in section 4 . The performance evaluation of proposed approach is described in section 5. A comparative analysis carried out between proposed and an earlier approach is also represented in this section. Finally, section 6 concludes the paper.

\section{Related work}

In general, the digital watermarking algorithms can be classified into two classes depending on the domain of watermark embedding. The first group belongs to the algorithms which use the spatial domain for data hiding $[5,6]$ while algorithms of the second group take advantage of transformation domains like discrete cosine transform (DCT) [7, 8], discrete Fourier transform (DFT) [9] and discrete wavelet transform (DWT) [10-15] for watermarking purpose. Though the spatial domain approaches are simple and less complex, the common drawback of the all the spatial domain image watermarking approaches is reduced security. Due to the image representation in its original format, the attacker can acquire the embedded data more easily. Further the previous works reveal that transform domain schemes are typically more robust to noise, common image processing and compression when compared with the spatial transform schemes [16]. In [11] a secure image watermarking scheme was proposed based on the integer wavelet transform and singular value decomposition. However the information loss is observed to be more in this approach due to the IWT. Further the proposed artificial bee colony (ABC) based image watermarking scheme in [12]. Here the $A B C$ algorithm was accomplished for the optimization of watermarking constant but the convergence of $\mathrm{ABC}$ is observed to be more. To achieve higher resistance over image processing attacks, a hybrid method was proposed in [13] based on DWT, FFT and SVD. Though this approach achieves good results for image processing attacks, this is not robust for geometric attacks like cropping, rescaling etc. A similar approach is developed in [14] by accomplishing the particle swarm optimization along with DWT and SVD. Here the PSO optimizes the watermarking constant, but not focused over the attacks which were not secure. Further these methods typically provide higher image imperceptibility and are much more robust to image manipulations, but the computational cost is higher than spatial domain watermarking methods. The performance of watermarking methods was further improved by combining two or more transformations [17].

The singular value decomposition (SVD) is extensively used in image watermarking field in recent years due to its features. However, various researchers pointed out the false positive detection problem in most of the SVD-based algorithms [18, $21,22,24]$. To counter this problem, numerous researchers have proposed improved versions of SVD-based image watermarking schemes. A robust image watermarking scheme based on SVD that embeds the entire watermark is given in [20]. There are two versions of this scheme depending on the implementation of SVD, to entire cover image and block-wise. The imperceptibility of an image watermarking scheme using block based SVD proposed in [19] is improved by incorporating compensation operation.

Though the features extracted are robust for so many attacks, the efficiency of watermarking approach also depends on the watermarking constant. There are so many approaches proposed in earlier towards the optimization of watermarking constant. PSO is an intelligent algorithm that using the stochastic, population-based computer algorithm for problem solving. Zheng [21] applied the PSO to search the embedding location of the integer DCT coefficients in a block to optimize the requirement of imperceptibility and robustness in watermarking. [22] and [23] utilized the PSO method to search for the optimal energy of embedding watermark to balance the quality and robustness of watermarked image. However the extracted watermark image through this approach contains a diagonal line due to the accomplishment of IWT.

\section{Preliminaries}




\subsection{Wavelet transform (WT)}

In DWT, a time scale representation of digital signal is obtained using digital filtering techniques. The signal to be analyzed is passed through filters with different cutoff frequencies at different scales. Each level of decomposition of DWT decomposes an image into four subbands, namely $L L, H L, L H$, and $H H$. The lower resolution approximation subband $(L L)$ that contains a rough description of the image is obtained by passing the digital signal through a low-low filter in both directions. The $H L$ and $L H$ subbands are obtained by passing through a low-pass filter in one direction and through a highpass filter in another direction. The $H H$ subband is high-pass filtered in both directions and contains the high-frequency components along the diagonals. After the image is processed by the wavelet transform, most of the information contained in the original image is concentrated into the $L L$ image. $L H$ contains mostly the vertical detail information which corresponds to horizontal edges. $H L$ represents the horizontal detail information from the vertical edges. DWT is very useful to determine areas in the original image where a watermark can be imperceptibly inserted because of its excellent Spatio-frequency localization properties. So, it is commonly used for watermarking. On the other hand, DWT has a lot of disadvantages. The major disadvantage is the shift variant. This occurs due to the down-sampling process after each level of filtering, which causes a significant change in the wavelet coefficients of the image even for minor shifts in it. This leads to inaccurate extraction of the watermark data and the cover image. RWT is established to overcome that problem because it is shift invariant.

\subsection{Singular value decomposition (SVD)}

SVD is an important tool in linear algebra, which is widely applied in many research fields such as principal component analysis, canonical correlation analysis and data compression. Let $\mathrm{X}$ denotes a matrix with size $\mathrm{M} \times \mathrm{N}$. The decomposition for $\mathrm{X}$ can be represented by Eq. (1),

$$
\begin{aligned}
X & =\left[\begin{array}{cccc}
X(1,1) & X(1,2) & \ldots & X(1, N) \\
X(2,1) & X(2,2) & \cdots & X(2, N) \\
\vdots & \vdots & \ddots & \vdots \\
X(M, 1) & X(M, 2) & \cdots & X(M, N)
\end{array}\right] \\
X & =U S V^{T}
\end{aligned}
$$

Where $\mathrm{U}$ and $\mathrm{V}$ components are composed of eigenvectors of matrix $\mathrm{X}$, and $\mathrm{T}$ represents the conjugate transpose operation. The $\mathrm{U}$ and $\mathrm{V}^{\mathrm{T}}$ components are called the left eigenvector and right eigenvector, respectively. The two components are also orthogonal matrices, which can be specified by Eq. (2),

$$
\begin{gathered}
I_{M}=U_{M}^{T} U_{M} \\
I_{N}=V_{N}^{T} V_{N}
\end{gathered}
$$

Where $I_{M}$ and $I_{N}$ are identity matrices with size $\mathrm{M} \times \mathrm{M}$ and $\mathrm{N} \times \mathrm{N}$, respectively. The component $\mathrm{S}$ is a singular value matrix in SVD domain, and is a diagonal matrix with non-negative real numbers,

$$
S_{M N}=\left[\begin{array}{ccccc}
\sigma(1,1) & 0 & \ldots & 0 \\
0 & \ddots & \cdots & 0 \\
\vdots & \vdots & \ddots & \vdots \\
0 & 0 & \cdots & \sigma(M, N)
\end{array}\right]
$$

Where $\sigma(1,1) \geq \sigma(2,2) \geq \cdots \geq \sigma(M, N) \geq 0$

\subsection{Genetic algorithm}

Genetic algorithm is one of the most widely used artificial intelligent techniques belonging to the area of evolutionary computation. Usually, a simple GA is mainly composed of three operations: selection, genetic operation, and replacement. Initially, a population is randomly generated. The fitness function then uses objective values from objective function to evaluate the fitness of each chromosome. The fitter chromosome has the greater chance to survive during the evolution process. The objective function is problem specific; its objective value can represent the system performance index (e.g., an error). Next, a particular group of chromosomes is chosen from the population to be parents. The offspring is then generated from these parents by using genetic operations, which normally are crossover and mutation. Similar to their parents, the fitness of the offspring is evaluated and used in replacement processes in order to replace the chromosomes in the current population by the selected offspring. The GA cycle is then repeated until a desired termination criterion is satisfied, for example, the maximum number of generations is reached or the objective value is below the threshold.

\section{Proposed method}

The complete details of the proposed approach are illustrated in this section. The proposed approach is accomplished in two phases, embedding phase and extracting phase. 


\subsection{Embedding procedure}

Here, the GA is used twice for the selection of watermarking constant alpha $(\propto)$. Generally, the singular values of LL sub-band are much more than the singular value of remaining sub-bands such as LH, HL and HH. So, two watermarking constants are derived through GA. They are designated as $\propto_{L L}$ and $\propto_{i}, i \in L H, H L, H H$ for embedding of singular values of LL sub-band of Host image (X) with singular value of LL band of watermark image (W) and the embedding of remaining bands respectively. Since, it is already revealed that, as the value of watermarking constant increases, it increases the robustness but decreases the quality. Also, the LL band having fewer variations whose effect will be less on the watermark, the watermarking constant $\propto_{L L}$ will be chosen as high compared to $\propto_{i}$. For both, GA gives the optimized value such that there will be a tradeoff between the robustness and imperceptibility. The details procedure of embedding is described below:

Step 1: Decompose the Host image (X) through RWT into the four sub-bands such as LL, LH, HL and $\mathrm{HH}$.

Step 2: Perform SVD for LL band and also for remaining bands as

$$
\begin{aligned}
& A_{L L}^{X}=U_{L L}^{X} S_{L L}^{X}\left(V_{L L}^{X}\right)^{T} \\
& A_{i}^{X}=U_{i}^{X} S_{i}^{X}\left(V_{i}^{X}\right)^{T}, i \in L H, H L, H H
\end{aligned}
$$

Step 3: Decompose the watermark image (W) through RWT into the four sub-bands such as LL, $\mathrm{LH}, \mathrm{HL}$ and $\mathrm{HH}$.

Step 4: Perform SVD for LL band and also for remaining bands as

$$
\begin{aligned}
& A_{L L}^{W}=U_{L L}^{W} S_{L L}^{W}\left(V_{L L}^{W}\right)^{T} \\
& A_{i}^{W}=U_{i}^{W} S_{i}^{W}\left(V_{i}^{W}\right)^{T}, i \in L H, H L, H H
\end{aligned}
$$

Step 5: Modify the singular values $\left(A^{X}\right)$ of every band of host image by embedding the singular values $\left(A^{W}\right)$ of every band of watermark image as

$$
\begin{aligned}
A_{L L}^{W I} & =A_{L L}^{X}+\propto_{L L} A_{L L}^{W} \\
A_{i}^{W I} & =A_{i}^{X}+\propto_{i} A_{i}^{W}, i \in L H, H L, H H
\end{aligned}
$$

Where $A_{L L}^{W I}$ are the singular values of LL band of watermarked image, $A_{i}^{W I}$ are the singular values of remaining bands of watermarked image. Here the $\propto$ value is optimized through the GA.

Step 6: apply inverse SVD on the altered singular values of all bands. The new bands are denoted as $W I_{L L}, W I_{L H}, W I_{H L}, W I_{H H}$

Step 7: The watermarked image is then obtained after applying the inverse RWT on the four sets of modified RWT coefficients.

$$
W I=R W T^{-1}\left(W I_{L L}, W I_{L H}, W I_{H L}, W I_{H H}\right)
$$

Where, WI represents the watermarked image.

\subsection{Extraction procedure}

Here, the extraction is applied to extract the watermark image. The main intention of RWT is to reduce the information loss. Here, the same RWT is applied on the distorted watermarked image denoted as $W I^{*}$. The same optimization procedure is carried out here through GA to find the efficient watermarking constant for both LL band extraction and remaining bands extraction. The complete procedure is described below:

Step 1: decompose the distorted watermarked image $W I^{*}$ through RWT into sub-bands such as LL, LH, $\mathrm{HL}$ and $\mathrm{HH}$.

Step 2: Perform SVD for LL band and also for remaining bands as

$$
\begin{aligned}
& A_{L L}^{W I^{*}}=U_{L L}^{X} S_{L L}^{X}\left(V_{L L}^{X}\right)^{T} \\
& A_{i}^{W I^{*}}=U_{i}^{X} S_{i}^{X}\left(V_{i}^{X}\right)^{T}, i \in L H, H L, H H
\end{aligned}
$$

Step 3: extract the singular values of all bands ( $A_{L L}^{W^{*}}$ and $A_{i}^{W^{*}}$ ) of watermark image form the singular values of distorted watermarked image as

$$
\begin{aligned}
& A_{L L}^{W^{*}}=\frac{\left(A_{L L}^{W I^{*}}-A_{L L}^{X}\right)}{\alpha_{L L}} \\
& A_{i}^{W^{*}}=\frac{\left(A_{i}^{W I^{*}}-A_{i}^{X}\right)}{\propto_{i}}, i \in L H, H L, H H
\end{aligned}
$$

Step 4: then the distorted bands will be obtained by performing SVD on the obtained singular values of all bands as $W_{L L}^{*}, W_{L H}^{*}, W_{H L}^{*}, W_{H H}^{*}$.

Step 5: Then the final watermark can be extracted by applying inverse RWT on the obtained distorted wavelet bands as 


$$
W^{*}=R W T^{-1}\left(W_{L L}^{*}, W_{L H}^{*}, W_{H L}^{*}, W_{H H}^{*}\right)
$$

Where $W^{*}$ is the extracted watermark.

Step 6. Evaluate peak Signal to Noise Ratio (PSNR) and Normalized Correlation (NC) between the original and extracted watermark images.

Step 7. Apply Genetic Algorithm in an iterative fashion until achieving the optimal fitness function. The fitness function is derived from $\mathrm{NC}$ as follows

$$
\text { fitness }=1-\text { Average }\left(N C_{j}\right)
$$

Where

$$
N C_{j}=\frac{1}{n_{\text {attack }}} \sum_{k=1}^{n_{\text {attack }}} N C\left(W, W_{j}^{*, k}\right)
$$

Where $W$ is original watermark and $W_{j}^{*, k}$ represents the extracted watermark through the proposed approach characterized by the position of the $\mathrm{j}_{\text {th }}$ particle. The smaller fitness value means the better robustness. Here, $n_{\text {attack }}$ signifies the number of attacks,

\subsection{Signature embedding procedure}

Step 1: Apply SVD to the modified LL band.

Step 2: consider the $\mathrm{U}$ and $\mathrm{V}$ matrices and generate a digest for both $\mathrm{U}$ and $\mathrm{V}$ through SHA-1 algorithm as

Digest $(\mathrm{U})=$ SHA-1 $(\mathrm{U})$

Digest $(\mathrm{V})=$ SHA- $1(\mathrm{~V})$

Step 3: perform bitwise XOR operation between Digest(U) and Digest(V) and name it as TMP1.

Step 4: Generate a secret key having same size of TMP1 and perform XOR operation between TMP1 and Secret key, name it as TMP2.

Step 5: choose first eight bits of the TMP2 as a digital signature.

Step 6: divide the modified LL band into $8 * 8$ blocks.

Step 7: Pick up eight blocks based on the secret key and perform SVD for all eight blocks.

Step 8: Consider the $U$ matrix $\left(U_{r, c}\right)$ of every block and round of the values in the $U$ matrix to less than or greater than to its nearest integer after multiplying with $10, \mathrm{U}_{\mathrm{r}, \mathrm{c}}^{\mathrm{m}}=\left\lfloor\mathrm{U}_{\mathrm{r}, \mathrm{c}}\right\rfloor$.
Step 9: Based on the digital bit stream $S G$, the $U_{\mathrm{r}, \mathrm{c}}^{\mathrm{m}}$ is modified as follows;

(a) If $U_{r, c}^{m}$ is even and the digital signature bit is 1 or if the $U_{r, c}^{m}$ is odd and the digital signature bit is 0 , then add one to the $U_{r, c}^{m}$.

(b) Otherwise keep $U_{r, c}^{m}$ unchanged.

Step 10: Perform inverse SVD for all the blocks and reconstruct the modified LL band.

\subsection{Signature extraction procedure}

Step 1: divide the LL band into $8 \times 8$ blocks.

Step 2: Select the blocks based on the secret key

Step 3: perform SVD for all the selected blocks

Step 4: Examine the $U_{r, c}$ through the following condition:

$$
\operatorname{sig}(i)=\left\{\begin{array}{lr}
1 & \bmod \left(\left\lfloor U_{r, c} \div 10\right\rfloor, 2\right)=0 \\
0 & \text { otherwise }
\end{array}\right.
$$

Where $i=1,2, \ldots, 8$ is the digital signature length.

\section{Simulation results}

The proposed waterarking apporach was implemented using MATLAB. To test the proposed apporach various test images are considreed and the size of host image is kept as $128 * 128$ and the watermark image $128 * 128$. The evaluation of the performance of proposed apporach under various circumstances was conducted in terms of imperceptibility and robustness against various attacks. The most widely used criteria are the peak signal-to-noise ratio (PSNR) and the normalized correlation (NC), which are employed consecutively. The PSNR is utilized to estimate the imperceptibility, a term used to evaluate the similarity between a host image and a water-marked image, and can be defined as follows:

$$
M S E=\frac{1}{M X N} \sum_{i=1}^{M} \sum_{j=1}^{N}\left(w(i, j)-w^{*}(i, j)\right)^{2}
$$

Where

$w=$ original watermark image

$w^{*}=$ extracted watermark image

$$
\begin{aligned}
& P S N R=10 \log \left(255^{2} / M S E\right) \\
& S S I M=\frac{\sum_{i} \sum_{i} w(i, j) \otimes w^{*}(i, j)}{\sum_{i} \sum_{i}(w(i, j))^{2}}
\end{aligned}
$$

Various types of attacks are accomplished over the 


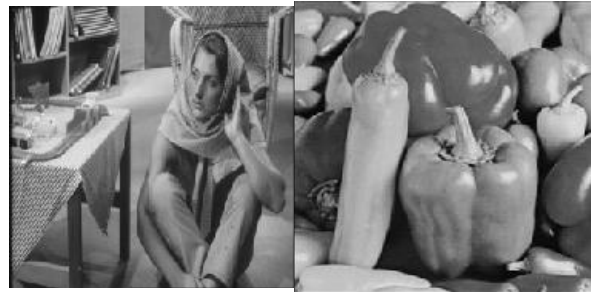

(a)

(b)

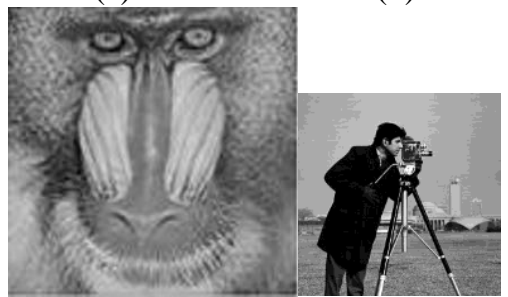

(c)

(d)

Figure.1 testing imagery (a) Barbara (b) peppers (c) baboon (d) camera mann

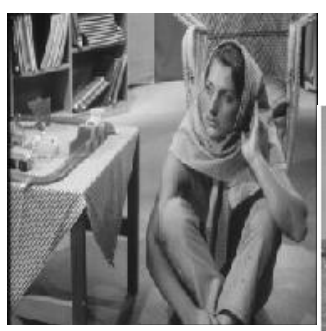

(a)

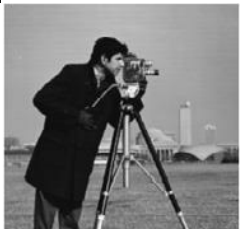

(b)
Figure. 2 obtained results under no attack secnario (a) Signed Watermarked image (b) Extracted Watermark

watermarked image to reveal the robustness of proposed scheme. To investigate the robustness of proposed approach, the watermarked image was subjected to eight attacks such as: (1) Gaussian noise Attack (GNA) (2) salt \& pepper noise attack (SPA) (3) Median Filtering attack (MFA), (4) Histogram Equalization attack (HEA), (5) Rotation attack (RA) (6) Contrast Enhancemnet attack (CEA) (7) cropping attack (CA) and (8) Scalling Attack (SA). The obtained results for both no attack and attack scenarios is represneted below.

In this test case, the signed watermarked image is not subjeced to any attack. The signed watermarked image is passed to extrcation unit as it is without any modification and for the extracted watermark, the performance metrics are evaluated.

In the case of attack scenario, the signed watermaekd image is subjeted to attacks and the it is passed to extrcation unit to reveal te performance of proposed approach. Further the obtained rsults of watermarked image and the extracted image are shown in the table.1. Here initially the Cameramann image is embedded in the Barbara image and the obtained PSNR, NC and the SSIM for both attack and no-attack cases are represneted in table.2. Similarly the Camremanna image is embedded in the baboon and peppers image and its respective results are represented Figs. 3 and 4.

Fig.3 illustrates the perfomrance of proposed approach under various test cases for a given Baboon host and Cameramann watermark image. The PSNR is observed to be high for the proposed apprach compared to the conventional one for the both attack a dno-attack scenarios. Since there is optimization at both fetaure extrcation leve and also atembedding phase, the quality of image is preserved in an efficient manner. Along with the PSNR, the NC also observed to be high for the proposed approach compared to the conventional apporach.Since the proposed apporach adopts a new signature based authentication mechanism the watermarked image is more robust to all types of atacks. Similarly Fig. 4 reveals the performance of proposed apporach for the test case of Peppers with cameramann image. For this case also the proposed approach is said to be achiveed better results compare to conventional approach.

\section{Conclusion}

In this paper, a new image waterarking approach was proposed based on redundant wavelet transform and Genetic Algorithm. The main objective of RWT is to reduce the information loss which is the main drawback with conventional discrete wavelet ransforms. GAis utilized to optimize the strength of watermarking constnat such that there should be a tradeoff between the robustness and the imperceptibility. Simulation is carreid out over various images and also over various attacks. An optimized alpha value is selected by considering all the attacks through GA algorithm. In this approach, the alpha optimization is carried out for two phases, one is for low varinat information (LL band) and another is for high varinat information (LH,HL and $\mathrm{HH}$ bands). The range of watermarking constnat derived through GA for LL band is high compared to the watermarking constant of remaining bands. The sumulation results also revealed that the proposed approach is robust for all types of atacks compared with conventional approach. On an average the proposed approach achieved an increment of $8.8175 \mathrm{~dB}$ and 0.0042 in the PSNR and NC respectively form the conventional DWT-SVDPSO approach.

This approach focused on the complete sub bands obtained afetr RWT for embedding. Though this approach achives an efficient reslience towards all types of attacks. The computational time is more. In future this problem can be solved by prospoing a 
Table 1. Observed results (watermarked image \& extracted watermark image)

\begin{tabular}{|c|c|c|c|c|}
\hline \multirow{2}{*}{ Scenario } & \multicolumn{2}{|c|}{ Results (Case 1) } & \multicolumn{2}{|c|}{ Results (Case 2) } \\
\hline & Watermarked & Extracted & Watermarked & Extracted \\
\hline $\begin{array}{c}\text { No } \\
\text { Attack }\end{array}$ & & & & \\
\hline GNA & & & & \\
\hline SPA & & & & \\
\hline MFA & & & & \\
\hline HEA & & & & \\
\hline RA & & & & \\
\hline CEA & & & & \\
\hline $\mathrm{CA}$ & & & & \\
\hline SA & & & & \\
\hline
\end{tabular}


Table 2. Performance metrics for the test case of Barbara and Cameramann Image

\begin{tabular}{|c|c|c|c|c|c|c|}
\hline \multirow{2}{*}{ Attack } & \multicolumn{3}{|c|}{ DWT-SVD-PSO [14] } & \multicolumn{3}{c|}{ RWT-SVD-GA } \\
\cline { 2 - 7 } & PSNR & NC & SSIM & PSNR & NC & SSIM \\
\hline NA & 44.9570 & 0.9971 & 0.9826 & 55.7328 & 0.9978 & 0.9912 \\
\hline GNA & 42.7314 & 0.9856 & 0.9649 & 52.1414 & 0.9899 & 0.9800 \\
\hline SPA & 42.9034 & 0.9959 & 0.9451 & 50.5246 & 0.9968 & 0.9590 \\
\hline MFA & 41.4260 & 0.9342 & 0.9416 & 50.0323 & 0.9387 & 0.9556 \\
\hline HEA & 30.9549 & 0.9262 & 0.9337 & 46.1825 & 0.9303 & 0.9448 \\
\hline RA & 25.8460 & 0.9756 & 0.8728 & 39.7327 & 0.9821 & 0.8903 \\
\hline CEA & 35.5280 & 0.9342 & 0.9327 & 44.6191 & 0.9456 & 0.9602 \\
\hline CA & 22.6259 & 0.9649 & 0.8556 & 30.7360 & 0.9727 & 0.8649 \\
\hline SA & 33.2743 & 0.9951 & 0.9635 & 38.7216 & 0.9970 & 0.9832 \\
\hline
\end{tabular}

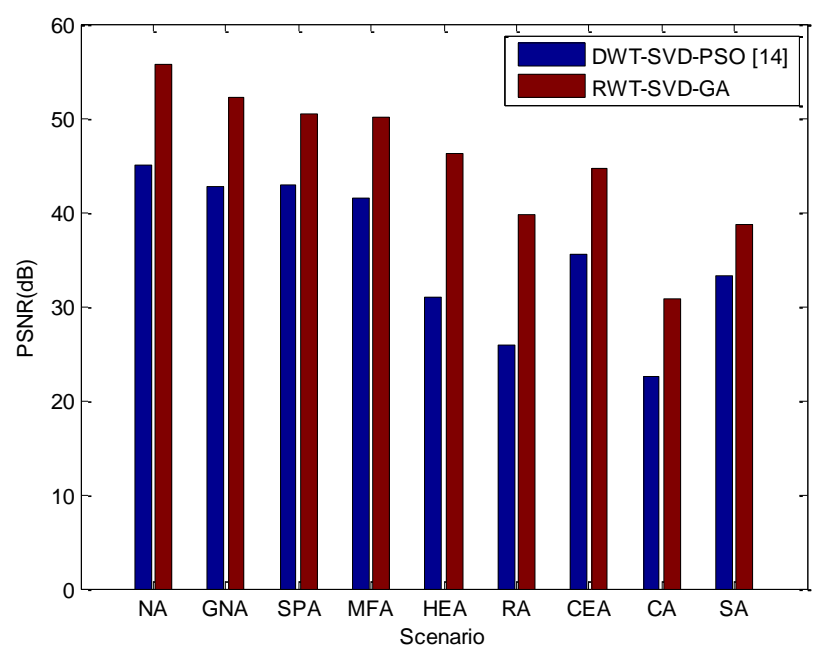

(a)

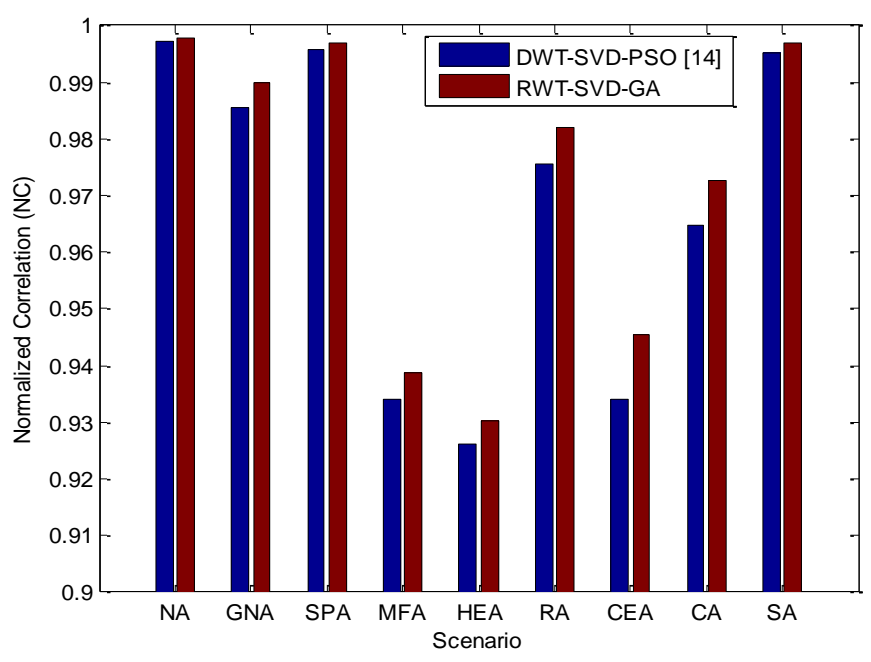

(b)

Figure.3 Comparitive analysis for the test of Baboon with Cameramann image with reference to (a) PSNR (b) NC

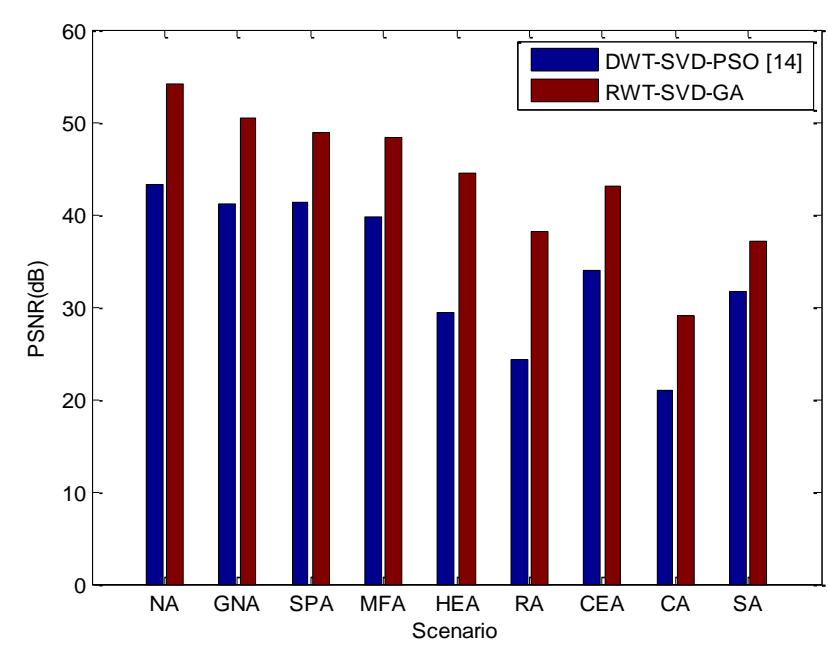

(a)

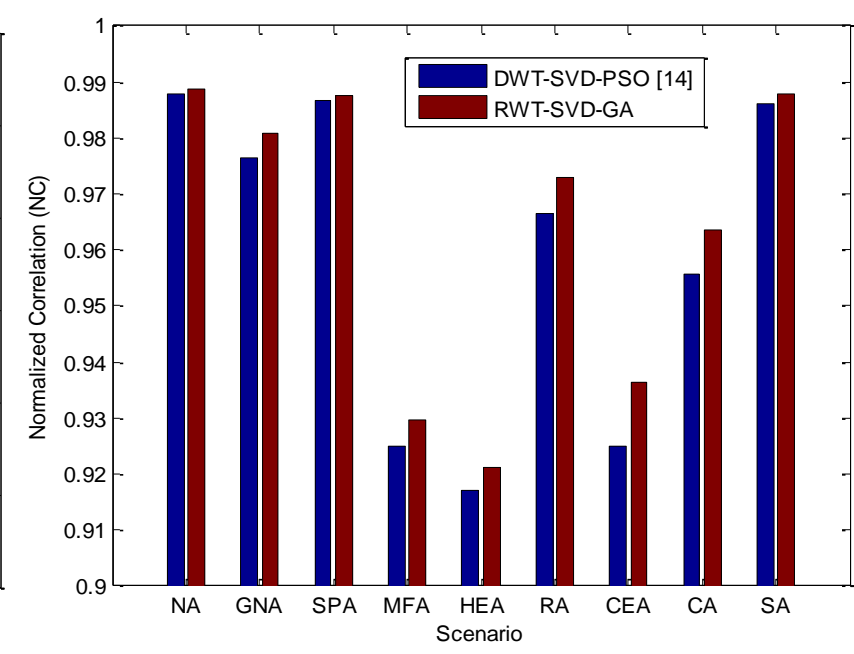

(b)

Figure.4 Comparitive analysis for the test of Peppers with cameramann image with reference to (a) PSNR (b) NC

sub band adaptive filter to extract only the required and optimal feature set for embedding. The Mean Square Deviation based sub band adaptive filtering can reduce the additional overhead incurred dueing the embedidng process.

\section{References}

[1] I. J. Cox, J. Kilian, F. T. Leighton, and T. Shamoon, "Secure spread spectrum watermarking for multimedia", IEEE Transactions on Image Processing, Vol. 6, No. 12, pp. 1673-1687, 1997. 
[2] W. Zeng and B. Liu, "A Statistical watermark detection technique without using original images for resolving rightful ownerships of digital images", IEEE Transactions on Image Processing, Vol. 8, No. 11, pp. 1534-1548, 1999.

[3] J. L. Liu, D.C. Lou, M.C. Chang, and H. K. Tso, "A robust watermarking scheme using selfreference image", Computer Standards \& Interfaces, Vol. 28, No. 3, pp. 356-367, 2006.

[4] T. Hai, C. M. Li, J. M. Zain, and A. N. Abdalla, "Robust image watermarking theories and techniques: A Review", Journal of Applied Research and Technology, Vol. 12, No. 1, pp. 122-138, 2014.

[5] I.G. Karibali and K. Berberidis, "Efficient spatial image watermarking via new perceptual masking and blind detection scheme", IEEE Transactions on Information and Forensics Security, Vol.1, No.2, pp.256-274, 2006.

[6] N. Nikolaidis and I. Pitas, "Robust image watermarking in spatial domain", Signal Processing, Vol.66, No.3, pp.385-403, 1998.

[7] T. Lan and A. Tewfik, "A novel high-capacity data-embedding system", IEEE Transactions on Image Processing, Vol.15, No.8, pp.24312440, 2006.

[8] G. L. Varco and W. Puech, "DCT-based data hiding for securing ROI of color images", In: Proc. of the IEEE International Conf. on Image Processing, pp. 1086-1089, 2005.

[9] F. Ahmed and I. S. Moskowitz, "A semireversible watermark for medical image authentication", In: Proc. of the $1^{\text {st }}$ Conf. on Distributed Diagnosis and Home Healthcare, pp. 59-62, 2006.

[10] H. T. Hu and L. Y. Hsu, "Exploring DWTSVD-DCT feature parameters for robust multiple watermarking against JPEG and JPEG2000 compression", Computers and Electrical Engineering, Vol. 41, No.1, pp. 5263, 2015.

[11] N. M. Makbol and B. E. Khoo, "A new robust and secure digital image watermarking scheme based on the integer wavelet transform and singular value decomposition", Digital Signal Processing, Vol. 33, pp. 134-147, 2014.

[12] M. Ali, C. W. Ahn, M. Pant, and P. Siarry, "An image watermarking scheme in wavelet domain with optimized compensation of singular value decomposition via artificial bee colony", Information Sciences, Vol. 301, No.4, pp. 4460, 2015.

[13] N. Tiwari, H. Naveen, and D. Goyal, "Improved Digital Image Watermarking
Algorithm Based on Hybrid DWT-FFT and SVD Techniques", Indian Journal of Science and Technology, Vol.10, No.3, pp.1-7, 2017.

[14] D. Sanku and S. Kiran, "Digital Image Watermarking in RGB Host Using DWT, SVD, and PSO Techniques", In: Proc. of Conf. on Micro-Electronics, Electromagnetics and Telecommunications, pp.333-342, 2018.

[15] P. Jaina and U. Ghanekar, "Robust watermarking technique for textured images", In: Proc. of International Conf. on Smart Computing and Communications, pp.179-186, 2017.

[16] A. Hanjalic, G. C. Langelaar, P. Vanroosmalen, J. Biemond, and R.L. Lagendijk, "Image and Video Databases: Restoration, Watermarking and Retrieval", IEEE Circuits and Devices Magazine, Vol.17, No. 4, pp.37-38, 2001.

[17] N. Liu, H. Li, H. Dai, D. Guo, and D. Chen, "Robust blind image watermarking based on chaotic mixtures", Nonlinear Dynamics, Vol. 80, No. 3, pp. 1329-1355, 2015.

[18] M. Q. Fan, H. X. Wang, and S. K. Li, "Restudy on SVD-based watermarking scheme", Applied Mathematics and Computation, Vol. 203, No. 2, pp. 926-930, 2008.

[19] A. A. Mohammad, A. Alhaj, and S. Shaltaf, "An improved SVD based watermarking scheme for protecting rightful ownership", Signal Processing, Vol. 88, No. 9, pp. 21582180, 2008.

[20] Q. Su, Y. Niu, H. Zou, and X. Liu, "A blind dual color images watermarking based on singular value decomposition", Applied Mathematics and Computation, Vol. 219, No. 16, pp. 8455-8466, 2013.

[21] Y. Zheng, C.H.Wu, Z. M. Lu, and W.H.lp, "Optimal robust image watermarking based on PSO and HVS in integer DCT domain", International Journal of Computer Sciences and Engineering System, Vol. 2, No.4, pp. 281287, 2008.

[22] E. Vahedi, C. Lucas, R. A. Zoroofi, and M. Shiva, "A new approach for image watermarking by using particle swarm optimization", In: Proc. of IEEE International Conf. on signal processing and communications, pp. 1383-1386.

[23] K.V.S. Reddy, "An Intelligent And Robust Digital Image Watermarking Scheme For Security Enhancement", IOSR Journal of Electronics and Communication Engineering, Vol. 12, No.5, pp. 45-54, 2017. 
[24] K.V.S. Reddy and J. Mounika, "Block Based Image Watermarking Through Wavelet Transform and Arnold Transform", International Journal of Innovative Technologies, Vol.04, No.20, pp.3855-3859, 2016. 